\title{
Evaluation of Small Amount Mobile Conditional Cash Transfers (mCCTs) to Improve Immunization Coverage and Timeliness
}

Subhash Chandir ${ }^{1,2}$, MBBS, MPH, PhD; Danya Arif Siddiqi ${ }^{1}$, MSc, BSc; Vijay Kumar Dharma ${ }^{1}$, MBBS, MPhil; Mubarak Taighoon Shah ${ }^{1}$, PharmD, MPhil; Aamir Javed Khan ${ }^{1}$, MD, PhD

${ }^{1}$ Interactive Research and Development, Karachi, Pakistan

${ }^{2}$ Harvard Medical School Center for Global Health Delivery-Dubai, Dubai, United Arab Emirates

\section{Corresponding Author:}

Subhash Chandir, MBBS, MPH, PhD

Interactive Research and Development

Woodcraft Building

Plot 3 \& 3-A, Sector 47, Korangi Creek Road

Karachi,

Pakistan

Phone: +971558507091

Email: subhashchandir@gmail.com

Harvard Medical School Center for Global Health Delivery-Dubai

Building 14

Dubai Healthcare City

Dubai,

United Arab Emirates

Phone: +971558507091

Email: subhashchandir@gmail.com

\begin{abstract}
Background: Globally, one in every five children remain unvaccinated, and over 2 million children die from vaccine-preventable diseases annually, primarily in resource-poor, low- and middle-income countries. The uptake of immunizations under routine Expanded Program on Immunization (EPI) is low due to limited geographical reach, poorly motivated staff, and parents' poor socio-economic status and opportunity cost associated with taking their child for immunization. Incentive-based approaches have been rigorously demonstrated to increase take-up and completion rates of immunization effectively. However, evidence from programs evaluating the impact of small-scale conditional cash transfers on immunization coverage is scarce.

Objective: This program aims to improve both immunization coverage and timeliness through the introduction of small mobile money-based Conditional Cash Transfers (mCCTs) for parents and performance-based incentives for vaccinators through widely available mobile money transfers. The outcome of interest is fully immunized child (FIC) coverage-i.e. a child who has received one dose of BCG and three doses of each OPV, Pentavalent \& PCV immunizations, and one dose of Measles vaccines. Outcomes will be compared through a baseline and endline immunization coverage survey to determine the effect of incentives.

Methods: The two-year program is implemented in a district of Sindh province in southern Pakistan in 34 government immunization centers with 86 vaccinators. Two different incentive schemes (per fully immunized child) are deployed to determine the minimum CCT amount required for follow-up. The total per child incentive for the high-incentive scheme is USD 10.5 and USD 5.0 for the low-incentive scheme. Parents/caregivers of children (0-24 months) visiting the immunization centers for any of the six routine vaccine visits are enrolled into a phone-based digital immunization registry. At enrollment, the child's bio-data and immunization history are recorded and a unique Quick Response (QR)-code sticker for identification is pasted on the child's immunization card. For the follow-up immunization visits, 3 SMS reminders are sent to parents for each immunization. At the follow-up visit, the immunization history of the child is pulled on the phone by scanning the QR-code, and following immunization, the child is randomly enrolled (with a 50\% probability) in either of the two incentive schemes. The mCCT is initiated automatically through the Registry which can be encashed through any of the mobile money retailers located in the area.
\end{abstract}

Results: The Program enrollments commenced on March 1, 2016. As of July 3, 2017, 43,418 children have been enrolled. Overall interim results show 59\% and 54\% age-appropriate coverage for Measles-1 and FIC (BCG+Pentavalent3+Polio3+Measles1) as compared to the baseline rate of $35 \%$ and $18 \%$ respectively. Disaggregating coverage rates by incentive schemes show $51 \%$ 
and 55\% rate for Measles 1 and FIC in the low-incentive arm, and 52\% and 55\% rate for Measles1 and FIC in the high-incentive arm.

Conclusions: Small scale incentives given as mCCTs induce positive behavioral change resulting in greater immunization coverage and compliance with immunization schedules. Concrete evidence regarding the impact of small-scale incentives on immunization coverage and timeliness (as opposed to the widely available literature on large CCTs) will be available at the end of the project.

(iproc 2017;3(1):e47) doi: 10.2196/iproc.8560

\section{KEYWORDS}

Conditional Cash Transfer; Immunization; Timeliness; Coverage

\section{Multimedia Appendix 1}

Full poster.

[PDF File (Adobe PDF File), 354KB-Multimedia Appendix 1]

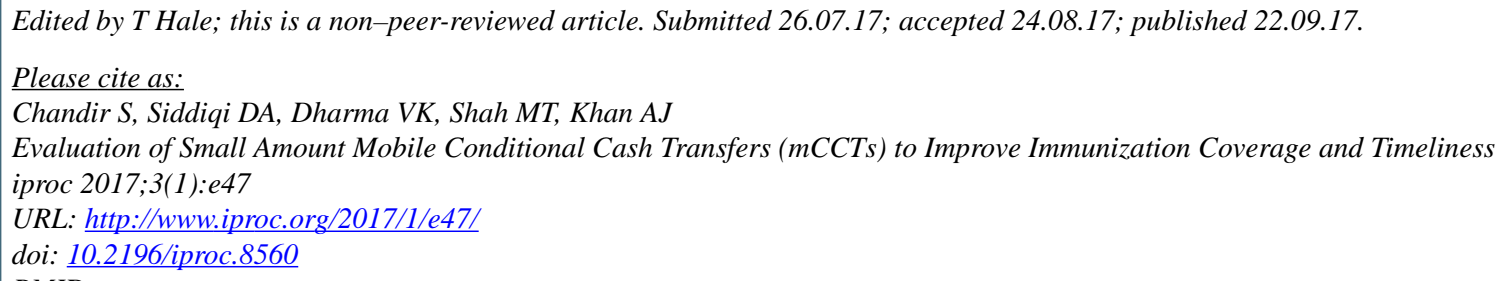

(C) Subhash Chandir, Danya Arif Siddiqi, Vijay Kumar Dharma, Mubarak Taighoon Shah, Aamir Javed Khan. Originally published in Iproceedings (http://www.iproc.org), 22.09.2017. This is an open-access article distributed under the terms of the Creative Commons Attribution License (https://creativecommons.org/licenses/by/4.0/), which permits unrestricted use, distribution, and reproduction in any medium, provided the original work, first published in Iproceedings, is properly cited. The complete bibliographic information, a link to the original publication on http://www.iproc.org/, as well as this copyright and license information must be included. 